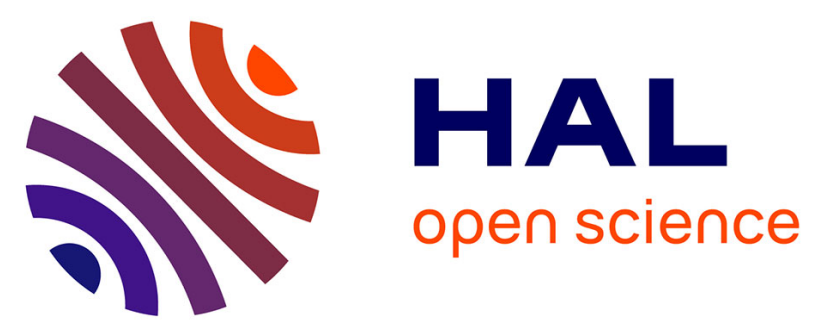

\title{
Simulation and experimental validation of a prototype electron beam linear accelerator for preclinical studies
}

Pierre Lansonneur, Vincent Favaudon, Sophie Heinrich, Charles Fouillade, Pierre Verrelle, Ludovic de Marzi

\section{To cite this version:}

Pierre Lansonneur, Vincent Favaudon, Sophie Heinrich, Charles Fouillade, Pierre Verrelle, et al.. Simulation and experimental validation of a prototype electron beam linear accelerator for preclinical studies. Physica Medica, 2019, 60, pp.50 - 57. 10.1016/j.ejmp.2019.03.016 . hal-03484577

\section{HAL Id: hal-03484577 \\ https://hal.science/hal-03484577}

Submitted on 20 Dec 2021

HAL is a multi-disciplinary open access archive for the deposit and dissemination of scientific research documents, whether they are published or not. The documents may come from teaching and research institutions in France or abroad, or from public or private research centers.
L'archive ouverte pluridisciplinaire HAL, est destinée au dépôt et à la diffusion de documents scientifiques de niveau recherche, publiés ou non, émanant des établissements d'enseignement et de recherche français ou étrangers, des laboratoires publics ou privés.

\section{(ㅇ)(1) $\$$}

Distributed under a Creative Commons Attribution - NonCommercial| 4.0 International 
Version of Record: https://www.sciencedirect.com/science/article/pii/S1120179719300560

Manuscript_2748e92b4b1dd11a087f1fd112a87501

Simulation and experimental validation of a prototype electron beam linear accelerator for preclinical studies

Pierre Lansonneur $^{1 *}$, Vincent Favaudon ${ }^{2}$, Sophie Heinrich ${ }^{3}$, Charles Fouillade $^{1,2}$, Pierre Verrelle $^{1,4}$, Ludovic De Marzi $^{1,2}$

${ }^{1}$ Institut Curie, PSL ResearchUniversity, Radiation OncologyDepartment, Proton Therapy Centre, Centre Universitaire, 91898 Orsay, France

${ }^{2}$ Institut Curie, University Paris Saclay, PSL ResearchUniversity, Inserm U 1021-CNRS UMR 3347, Centre Universitaire, 91405

Orsay, France.

${ }^{3}$ Institut Curie, RadeXpPlatform, Centre Universitaire, 91405 Orsay, France.

${ }^{4}$ Institut Curie, PSL Research University, Inserm U 1196-CNRS UMR 9187, Centre Universitaire, 91405 Orsay, France.

*corresponding author. E-mail address: kinetron_simulation@curie.fr

\section{ABSTRACT:}

Purpose: Measurements and Monte-Carlo simulations were carried out to model the dose distribution of a prototype electron beam linear accelerator (Kinetron LINAC) to determine the dose to organs in small animal irradiations experiments. Dose distributions were simulated using the GATE8.0/Geant4.10.3 Monte-Carlo platform, and measured in air and solid water phantoms using a commercial scintillating screen detector and new EBT-XD Gafchromic films.

Methods: The LINAC is able to produce 4.5 MeV electron beams at dose-rates ranging from Gy/min to thousands of Gy/s, and is used to study the radiobiological effects of very-high dose-rates that have been shown to protect normal tissues from radiation toxicity. Numerical simulations and experimental dosimetric characterisation of this electron accelerator were performed with the Monte-Carlo toolkit and various detectors. Absolute dose distributions in solid water were measured and compared with simulations. Realistic electron irradiation conditions were simulated in voxelised mice CT images. 3D dose distributions and dose-volume histograms in lungs of mice were simulated and analyzed.

Results: Measured and calculated depth-dose profiles for several beam configurations (energy and dose-rate) were compared. Beam emittance was validated by comparing measured and calculated beam sizes along the central axis in air: the deviation for all conditions was less than $1 \mathrm{~mm}$. A good agreement was obtained between experimental dose distributions and the results obtained with simulations ( $<2 \%$ dose differences for lateral and depth-dose profiles).

Conclusions: The method presented here, relying on few free parameters, can be adapted to very-high dose-rate electron irradiation to support the analysis of preclinical research experiments.

Keywords: FLASH, electron dosimetry, Monte-Carlo, Geant4.

\section{Introduction}

Normal tissue protection from radiation-induced toxicity is a major concern in radiation therapy, and the radiobiological effectiveness of irradiation is highly dependent on dose and dose-rate. In particular, some elements indicate that the radiobiological response of tissues to very short or pulsed irradiations may be poorly elucidated. Recent in vivo investigations have shown that very-high dose-rate (FLASH) irradiation with electrons was less harmful for healthy tissues (lungs) in mice, but just as effective as conventional dose-rate gamma rays in terms of tumour growth inhibition [1]. Similar protection of normal tissue in a FLASH experiment has also been recently demonstrated in brain [2]. This sparing effect on healthy tissues, without compromising the anticancer effect, would open up extremely interesting prospects in clinical radiation oncology. The emergence of a new generation of particle therapy facilities (very-high energy electron beams, laser-based accelerators [3, 4, 5]) is also leading to the use of instantaneous dose-rates or doses-per-pulse up to several orders of magnitude greater than those produced by conventional radiation sources. In order to assess the dose distributions received by small animals irradiated under FLASH conditions, and to create a predictive dosimetry tool, we hereby describe the Monte-Carlo simulation and validation of the $4.5 \mathrm{MeV}$ electron irradiation facility described in [1]. Such irradiators are gaining increasing attention following the 
demonstration of the FLASH sparing effect. It is also noteworthy that clinical LINACs can be turned into FLASH irradiators after simple modifications, thus paving the way to human treatments $([6,7])$.

Ionisation chambers, recommended in many clinical practice guidelines, are the cornerstone of reference dosimetry in radiation therapy. However, for pulsed radiation with high dose-per-pulse (more than $5 \mathrm{mGy}$ par pulse), incomplete charge collection due to recombination, space charge and ion drift effects requires the use of complex non-linear saturation correction factors. Current methods used for dosimetry at conventional dose-rates function poorly at pulsed very-high dose-rates [8, 9]. Dosimetric measurement is also a common issue in the case of very small field electron beams, such as those used for preclinical investigations [10]. An alternative is to use chemical dosimeters operating at sub-microsecond timescales, such as those used in pulsed electron radiolysis and based on the formation of methylviologen radicals ([11, 12])or radiochromic films [13]. Radiochromic films are extensively used in electron beam dosimetry because of their low dose-rate and energy dependence [8, $14,15]$.To obtain parameterisation and validation of our model, we therefore used EBT-XD Gafchromic films and a scintillating screen coupled to a CCD camera for relative dosimetry of FLASH fields, cross-calibrated to reference dosimetry using an ionisation chamber to measure the absolute absorbed dose under conventional dose-rate conditions.

\section{Methods and materials}

\section{LINAC specifications}

FLASH irradiation was performed using the linear electron accelerator (Kinetron LINAC) from CGR-MeV described in [1, 16]. This accelerator provides a beam with $4.5 \mathrm{MeV}$ nominal energy that can be operated according to two modes: a conventional irradiation mode (CONV), in which the mean dose-rate is set below $0.03 \mathrm{~Gy} / \mathrm{s}$, and a FLASH irradiation mode, in which typical instantaneous dose-rates up to $2 \times 10^{7} \mathrm{~Gy} / \mathrm{s}$ can be achieved. Time structures of the pulses for the two modes are listed in table 1 . The machine also allows the possibility to tune the beam energy between 4 and $5 \mathrm{MeV}$ by setting the magnetron voltage ("DQing" parameter). The Kinetron accelerating guide is powered by a magnetron (3-GHz band, $2.5 \mathrm{MW}$ peak power). The triode electron gun (11 kV accelerating potential) allows easy and reliable instant adjustment of beam current (10-250 mA) and pulse width (0.05-2.7 ps). The pulse-to-pulse reproducibility obtained is better than $5 \%$ [16].

\begin{tabular}{l|c|c} 
& FLASH mode & CONV mode \\
\hline Energy & 4 to $5 \mathrm{MeV}$ & 4 to $5 \mathrm{MeV}$ \\
Pulse duration & 0.1 to $2.2 \mu \mathrm{s}$ & $3 \mu \mathrm{s}$ \\
Repetition rate & 10 to $200 \mathrm{~Hz}$ & 10 to $200 \mathrm{~Hz}$ \\
Dose per pulse & $1 \mathrm{~Gy}$ & $1 \mathrm{mGy}$
\end{tabular}

Table 1. Beam pulse structure in FLASH and conventional (CONV) mode.

Fig. 1. Schematic view of the setup used for mice irradiation under FLASH conditions. 1, electron source. 2, high density polyethylene insulator. 3, LINAC front face. 4, primary graphite collimator ( $22 \mathrm{~mm}$ thick, $40 \mathrm{~mm}$ in diameter aperture). 5, $120 \times 120 \mathrm{~mm}$ secondary graphite collimator (18 x $26 \mathrm{~mm}^{2}$ rectangular aperture). 6 , position of irradiated mice. 7, EBT-XD films used to measure the dose distribution downstream and upstream from the mouse.

As depicted in Figure 1, two collimators made of $22 \mathrm{~mm}$ thick graphite are used to delimit the irradiation field, and protect the animals from whole-body exposure to radiation [1]. Time-resolved monitoring of the electron fluence is performed by measuring the charges collected in the first graphite collimator, electrically isolated by a high density polyethylene insulator. This plastic insulator is placed between the source and the first graphite collimator to allow real-time measurement of the beam current. The second graphite collimator is located $35 \mathrm{~mm}$ in front of the mouse with an $18 \times 26 \mathrm{~mm}^{2}$ rectangular aperture (for lung irradiation). The distance between the mouse and the electron source is $500 \mathrm{~mm}$.

\section{Monte Carlo modelling}

Monte Carlo simulations of photon irradiators have been extensively explored due to growing interests in radiosurgery over the last 40 years [17]. However, only few studies have been published about the modelling of electron LINAC for small animals [18, 19]. Simulations using the Gate 8.0 - Geant4.10.3 Monte-Carlo platform [20, 21] were performed to model the dose distribution of the beam and treatment head accelerator along the beamline. The physics builder set "emstandard_opt3"was used for electromagnetic interactions. The beamline components, such as the primary source, the insulator and the collimators, were modelled. The uncertainty was defined as the average statistical uncertainty in voxels with a dose greater than $90 \%$ of the maximum dose and was estimated from the square root of the average variance of the voxels. A total of $10^{9}$ electron histories 
were simulated for each setup such that the relative statistical uncertainty was less than $1 \%$. Simulations were run on a multiprocessor Linux cluster (64 bit architecture, 16 cores). The range cut for all particles was set to $0.5 \mathrm{~mm}$ in the mouse volume, corresponding to a production threshold of approximately $200 \mathrm{keV}$ for electrons. The dose scoring grid resolution was $0.5 \times 0.5 \times 0.5 \mathrm{~mm}^{3}$. All other parameters used default options.

\begin{tabular}{lll} 
Measurement & Dosimetric device & Parameter investigated \\
\hline Beam profile & LYNX, EBT-XD & $\mathrm{r}_{\mathrm{S}}, \sigma_{\ominus}$ \\
Depth-dose distribution (FLASH) & EBT-XD & $\mathrm{E}, \sigma_{\mathrm{E}}$ (FLASH) \\
Depth-dose distribution (CONV) & Ionisation chamber & $\mathrm{E}, \sigma_{\mathrm{E}}(\mathrm{CONV})$
\end{tabular}

Table 2. Experimental configurations used to set the beam source parameters.

The source was modelled in the simulation by a planar disk with radius $r_{s}$ oriented towards the $z$ axis. It delivers an electron beam with an energy $E$ and an energy spread $\sigma_{\mathrm{E}}$. Beam divergence is modelled by a Gaussian distribution with an angular spread $\sigma_{\ominus}$. Parameter optimisation was performed using the non-linear least squares method provided by the python scipy environment [22] in order to obtain a good agreement between measurements and simulated data (see Table 2).

\section{EBT-XD film dosimetry}

For an experimental validation of MC simulations, we used a new type of radiochromic films (EBT-XD, Ashland Inc., USA). The sensitivity, anisotropy and stability of this detector has been improved compared to previous models (eg EBT2-3), making it a good candidate for FLASH relative dosimetry [23]. Absolute dose-response of the EBT-XD Gafchromic films up to 40 Gy was assessed by cross-calibrating the optical densities of a series of films with an ionisation chamber calibrated in conventional mode.

The PPC05 parallel-plate ionisation chamber (IBA dosimetry, Germany) was used for absorbed dose-to-water determination, according to the IAEA TRS 398 recommendations for electron beam dosimetry. The absorbed dose-to-water was measured with a at the centre of an $8 \mathrm{~cm}$ diameter beam, in a water phantom located $78 \mathrm{~cm}$ away from the first graphite collimator. Polarity and recombination effects lower than $2 \%$ were observed with this chamber, and a beam quality correction factor ka of 0.936 was used, as measured by [24].

In order to compare the dose profiles measured by EBT-XD dosimetry with the ionisation chamber, a calibration curve was then performed for conversion of net optical density (netOD) to dose. The films were scanned 36 hours after exposure with an EPSON Expression 10000XL scanner with no corrections applied to the image, while keeping the film angular orientation constant. To avoid discrepancies, films from the same batch (lot $N^{\circ}$. 08021701) were used. The pixel values $P V_{\text {unexp }}$ and $P V_{\exp }$ of the unexposed films and films exposed to irradiation were then averaged over three independent measurements in order to compute the net optical densities (netOD) in each channel (R, $G$ and $B)$ :

$$
n e t O D=\log _{10}\left(\frac{\left\langle P V_{\text {unexp }}\right\rangle}{\left\langle P V_{\text {exp }}\right\rangle}\right)
$$

For each channel, the calibration curve was then fitted by the expression:

$$
D=a \cdot n e t O D+b \cdot n e t O D^{c}
$$

as proposed in reference [25] for EBT3 films, with D being the dose determined from the ionisation chamber reading and $a, b$ and $c$ the fitting parameters. Parameter optimisation was performed using the non-linear least squares method provided by the python scipy environment [22].

\section{Depth-dose distributions}

Schematic view of the setup used to measure the depth-dose distributions in conventional and FLASH modes is shown in Figure 1.1 left and right, respectively.

Fig 1.1. Schematic view of the setup used to measure the depth-dose distributions in conventional (left) and FLASH modes (right). Details are given in the text.

\section{Conventional dose-rate (CONV)}


To measure the depth-dose profiles under conventional conditions, a $50 \mathrm{~V}$ voltage was applied to the electron gun grid and the dose was delivered by $3.5 \mu$ s pulses delivered at a $200 \mathrm{~Hz}$ repetition rate $(2.1 \mathrm{mGy} / \mathrm{pulse})$. Three measurements with a PPC05 chamber placed inside a RW3 plastic phantom (IBA dosimetry, Germany) were averaged for each depth (experimental data are provided in Appendix 3). In order to overcome potential small variations of fluence between measurements, the chamber reading was cross-referenced to independent reading of a small cylindrical chamber used as a reference monitoring chamber. $A$ $1.76 \mathrm{~mm}$ offset corresponding to the front window of equivalent thickness ( $1 \mathrm{~mm}$ Shonka C552, density 1.76) was applied in order to take into account the effective point of measurement of the detector.

\section{Very high dose-rate (FLASH)}

In order to measure the depth-dose distribution under FLASH conditions (the use of an ionisation chamber is not suitable at such dose rates because of strong recombination effects), the EBT-XD film was placed horizontally between two RW3 slabs tightened with clamps and positioned $10 \mathrm{~cm}$ from the first collimator. To avoid underdosage artefacts at the entrance, an ultrasound transmission gel and another film sample were applied on the lateral side of the film at the entry surface of the phantom, as recommended by reference [26].

The dose was delivered at a dose-rate of $10^{6} \mathrm{~Gy} / \mathrm{s}(0.5 \mathrm{~Gy}$ pulses each delivered for $0.5 \mu \mathrm{s})$ at an expected energy of $5 \mathrm{MeV}$. The film was subsequently scanned with a resolution of $150 \mathrm{dpi}$ and pixel values were converted to dose units using equations (1) and (2) with the fit parameters of red channel.

\section{Geometric properties}

Beam profiles were measured with the LYNX (FIMEL, France), a $0.4 \mathrm{~mm}$ thick scintillator screen coupled to a charge-coupled device (CCD) camera. The field of view of the camera covers a $300 \times 300 \mathrm{~mm}^{2}(600 \times 600$ pixels) active area with an effective spatial resolution of $0.5 \mathrm{~mm}$. The detector has been previously characterised (dose linearity, dose-rate independence, spatial resolution) and has been reported to be a good candidate for charged particle beam dosimetry [27]. 2D dose distributions at various positions along the beam axis as well were obtained with the detector in order to evaluate the divergence and size of the electron source.

\section{Imaging, HU calibration and small animal irradiation}

In order to assess the dose deposited in a digital phantom, CT images of a mouse were acquired on a Small Animal Radiation Research Platform (SARRP, Xstrahl)[28], using the parameters listed in Table 3. As small animal image acquisition and irradiation cannot be carried out using the same equipment, a mouse was anesthetised, immobilised in a dorsal and vertical position, placed in a positioning device and kV-CBCT images were acquired. The stoichiometric calibration procedure described in [29] was used to determine the tissue substitute calibration curves of the SARRP, using a set of materials with known elemental composition and physical density close to those of tissue samples. This calibration was performed at the same time as mouse imaging, under equivalent geometrical conditions in order to mimic small animal scattering conditions. The calibration curve used to convert CT numbers into mass density is shown in Figure 2. The images were then converted into a voxelised digital phantom using the "Hounsfield Material Generator" implemented in the GATE toolkit, according to the procedure described in references [29, 30]. The small animal was then transferred to the irradiator facility, where irradiation was performed with films placed upstream and downstream from the mouse as shown in Figure 1 (the lungs of the mice were exposed to a $17 \mathrm{~Gy}$ bilateral thorax irradiation). kV-CBCT images were acquired again at the end of the irradiation to check for consistency in the animal position.

\begin{tabular}{l|l} 
Energy & $60 \mathrm{kV}$ \\
Number of projections & 720 \\
Grid resolution & $0.245 \times 0.245 \times 0.245 \mathrm{~mm}^{3}$
\end{tabular}

Table 3. Parameters used for $\mathrm{CBCT}$ acquisition and reconstruction.

Fig 2. Calibration curve used to convert CT numbers into mass density, according to the method described in reference [29, 30]. 
III. Results

\section{Dose-response curves of EBT-XD films}

The dose plotted as a function of the optical density of the films is shown in Figure 3, together with the fitting curve for a $5 \mathrm{MeV}$ irradiation.

Fig. 3. Dose-response curves of EBT-XD films under conventional dose-rate irradiation with $5 \mathrm{MeV}$ electrons. The optical density (netOD) is calculated using equation (1) as the average of three irradiations (points). Equation ( 2 ) is used to fit the response curve in each channel (dotted lines).

The fitting parameters corresponding to the dose-response curve shown in Figure 3 are listed in Table 4.

\begin{tabular}{c|ccc} 
Channel & $a(k=2)$ & $b(k=2)$ & $c(k=2)$ \\
\hline$R$ & $29.2 \pm 1.7$ & $145 \pm 8$ & $2.9 \pm 0.14$ \\
G & $53.5 \pm 3.7$ & $145 \pm 13$ & $2.3 \pm 0.2$ \\
B & $83.8 \pm 35.0$ & $395 \pm 46$ & $1.6 \pm 0.2$
\end{tabular}

Table 4. Fitting parameters for a coverage factor $k=2$ and associated error (1- $\sigma)$ for the dose-response curve shown in Figure 3. The blue channel presented the largest error and was not selected for dosimetric purposes in this study.

\section{Depth-dose distribution at conventional dose-rate (CONV)}

The depth-dose distribution of electrons measured in the RW3 plastic phantom with a PPC05 ionisation chamber under conventional conditions $(0.28 \mathrm{~Gy} / \mathrm{s})$ is shown in Figure 4. The dose distributions for the $5 \mathrm{MeV}$ electrons simulated for several energy spreads are shown in grey.

Fig. 4. Depth-dose distribution of electrons measured in a plastic phantom with a PPC05 ionisation chamber under conventional conditions. The dose distributions of $5 \mathrm{MeV}$ electrons simulated for different energy spreads are shown in grey.

The distribution with the best fit to the data corresponds to a relative energy spread $\sigma_{E} / E=0.27 \pm 0.03(k=2)$.

\section{Depth-dose distribution at very high dose-rate (FLASH)}

Figure 5.a shows the depth-dose distributions of $5 \mathrm{MeV}$ electrons measured with EBT-XD films under FLASH conditions, compared to $\mathrm{MC}$ simulations. It is noteworthy that the experimentally measured energy was consistent with the expected energy selected from the LINAC. The distribution with the best fit to the data corresponds to a relative energy spread $\sigma_{E} / E=0.06$ $\pm 0.01(\mathrm{k}=2)$. Monte-Carlo simulation was validated by comparing an independent depth-dose profile (measured for $4 \mathrm{MeV}$ set on the machine) with the output of the simulation. As shown in Figure 5.b, a good agreement $\left(\sigma_{E} / E=0.06 \pm 0.03, k=2\right)$ was observed between the Monte-Carlo simulation and the experimental curve.

Fig. 5. Depth-dose distributions of $5 \mathrm{MeV}(\mathrm{a})$ and $4 \mathrm{MeV}$ (b) electrons measured with the EBT-XD films in a plastic phantom. The simulated (MC) distributions are plotted as a dotted line.

In order to specify the energy and energy spread in the simulation, the depth-dose distribution was simulated for various pairs of parameters $\left(E, \sigma_{E}\right)$ and the distance to the experimental points (Chi-square) was then calculated for each pair, as shown in Figure 6.a.

Fig. 6. Chi square calculated for different pairs of parameters. (a) Energy and energy spread. (b) Source radius and beam divergence. The white line delimits the $95 \%$ confidence interval.

\section{Geometric properties}

Two free parameters may impact the electron beam size: the source radius and the angular spread at the exit of the source. In order to properly set their values in the simulation, beam sizes were measured at different locations inside the accelerator treatment head and along the beam axis. EBT-XD films were used for distances inside (between the source and the collimator) 
and close to the accelerator head, while the LYNX was used $0.9 \mathrm{~m}$ upstream from the collimator. Measurements therefore covered a region larger than $1 \mathrm{~m}$ in depth. The profiles obtained were fitted by a two-dimensional Gaussian distribution in both cases. The beam profile was simulated for various pairs of parameters $\left(r_{s}, \sigma_{\ominus}\right)$ and the chi square was then calculated for each pair as shown in Figure 6.b.

The spreads measured are shown in Figure 7 along with the fitted Monte-Carlo simulation for a source radius of $1.5 \mathrm{~mm}$ and an angular divergence of $13.5 \mathrm{deg}$.

Fig. 7. Beam profile, measured at several distances along the beam axis with (blue points) EBT-XD films and scintillating screen and (grey line) simulated data for a source radius $r_{S}=1.5 \mathrm{~mm}$ and beam divergence $\sigma_{\ominus}=13.5 \mathrm{deg}$.

The parameters (presenting the best fit with the data) used to model the electron LINAC are listed in Table 4.

\begin{tabular}{l|l} 
energy & $4.5 \pm 0.05 \mathrm{MeV}$ \\
rel. energy spread & $0.06 \pm 0.01$ (FLASH) \\
& $0.27 \pm 0.03$ (CONV) \\
angular spread & $13.5 \pm 1 \mathrm{deg}$ \\
source radius & $1.5 \pm 1 \mathrm{~mm}$
\end{tabular}

Table 4. Parameters used for source modelling together with their $95 \%$ confidence intervals $(k=2)$.

\section{Lung irradiation}

In order to validate the Monte-Carlo simulation in a complex geometry (dose heterogeneity in the lungs) and to assess the dose distribution in a typical preclinical study, we then compared the dose distribution measured on the films with the output of the simulation.

The dose distributions measured on the films placed upstream and downstream from the mouse are shown in Figure 8.a and Figure 9.a, respectively. The MC-generated doses (Fig. 8.b and 9.b) were normalised to the dose measured on the film placed upstream and simulated and measured images for each film were compared by $2 \% / 2 \mathrm{~mm}$ gamma index analysis [31] (Fig. 8.c and 9.c). For the film placed downstream from the mouse, on which large heterogeneities were observed, $90 \%$ of the pixels had a gamma index less than one.

A good agreement was also observed between the two dose profiles measured along a median line (white dotted line), as shown in Figure8.c and 9.c. The remaining discrepancy between the two distributions can be attributed to small movements of the mouse (despite being anaesthetised) between the CBCT acquisition and irradiation.

Fig. 8. Dose distributions on the film placed in front of the mouse, measured with the EBT-XD film (a) andMC-generated (b). The $2 \% / 2 \mathrm{~mm}$ gamma index is shown on the top-right corner $(\mathrm{c}) .91 \%$ of the pixels have a gamma indexless than one. The dose profiles measured along the white dotted line are shown on the bottom plot (d).

Fig. 9. Dose distributions on the film placed at the back of the mouse, measured with the EBT-XD film (a) and MC-generated (b). The $2 \% / 2 \mathrm{~mm}$ gamma index is shown on the top-right corner (c). $90 \%$ of the pixels have a gamma index less than one. The dose profiles measured along the white dotted line are shown on the bottom plot (d).

Monte-Carlo simulations can be used as a predictive tool to infer the dose received by irradiated organs. The dose distribution obtained when irradiating mouse lungs with the setup described in Figure 1 (corresponding to the experimental conditions of [1]) is shown in Figure 10. We first compared the dose distributions obtained between the conventional and FLASH irradiation modes of the machine. No significant differences were observed between the two distributions. However, in both modes, the difference between the doses received by the two lungs, due to the presence of the heart, was especially visible in the transverse and coronal planes. The difference in mean dose visible on the dose-volume histograms (Figure 10.c) was of the order of $10 \%\left(D_{50}\right)$.

Fig. 10. Monte-Carlo generated dose distribution and dose-volume histograms for mouse lung irradiation. (a), (b) and (c) represents the output for irradiation from the thorax, while irradiation from the back is shown on panels (d), (e) and (f). When 
irradiating from the thorax, the electron beam first passes through the heart located in front of the left lung, thereby inducing a significant difference of the dose received by the two lungs. Irradiating from the back results in a more homogeneous dose distribution in both lungs.

\section{Discussion}

The simulation presented here only focused on small animal irradiation and 3D dose distributions with millimetric voxel sizes. Since other parameters were fixed by the geometry of the setup, the limited number of free parameters needed for the simulation allows this methodology to be applied to other electron irradiation facilities. The detailed characteristics of the beam may also serve as input to more sophisticated modelling, accounting for interactions of electrons at the cellular/molecular scale that require substantial calculation time. Simulating the processes at such a scale will undoubtedly be relevant for a better understanding of the FLASH sparing effect, and could be implemented in future work within the Monte-Carlo toolkit such as Geant4-DNA. The physicochemical and recombination processes occurring at high dose rates were not considered in this study, as these features are currently not fully covered in the Geant4 Monte Carlo simulation toolkit. Once validated, these features could expand the range of applications of the modelling presented here.

In Geant4, the range cut value is converted into threshold energies for each material and for each particle type (i.e. electron, positron and gamma), so that the particle with threshold energy stops (or is absorbed) after travelling the range cut distance [32]. In the case of small animal radiation therapy, dose distributions with electron production cuts between $10 \mu \mathrm{m}$ and $1.0 \mathrm{~m}$ were compared [33], showing that a small overestimation in bone could be expected at the water/bone interfaces (partly due to the reduction of beam energy from MV to kV range for small animals). As no major discrepancies and no such differences at the water/lung interfaces or in homogeneous phantoms can be observed (same depth dose curves and mean doses within $2 \%$ ) with the distributions presented in this paper, we decided to use a $0.5 \mathrm{~mm}$ setting (that corresponds to a threshold energy of 200 $\mathrm{keV}$ for electrons) in order to reduce computation times. The $2 \mathrm{~mm}$ criterion for gamma index analysis originates from the uncertainty of the positioning reproducibility of the mouse with our setup and respiratory motion (up to millimetres for sites within the thorax). Several references also describe mouse irradiators for which the distance-to-agreement acceptance criterion used for the gamma function is $3 \mathrm{~mm}$ [34] and discuss the implications of respiratory motion for small animal image-guided radiotherapy [35].

A significant difference in the dose received by the two lungs was observed when comparing irradiations with an anterior or posterior beam (Fig. 10, left and right panels, respectively). This difference, attributed to shielding by the heart or spine, is minimised by irradiating the mouse from the back. The dose distribution obtained in such a configuration is displayed in Figure 10.d and 10.e. Although the spine may induce a slight underdosage in the right lung, the dose distribution is more homogeneous. This observation is confirmed on the dose-volume histograms shown in Figure 10.f. Relative doses D98 (corresponding to $98 \%$ irradiation of the left lung volume) of $52 \%$ and $66 \%$ were observed for anterior and posterior irradiation, respectively. The $\mathrm{D}_{50}$ dose difference between the two lungs also reached $10 \%$ for anterior irradiation and $5 \%$ for posterior irradiation, and these specific characteristics may be of interest when performing lung irradiation.

MC simulations are generally accepted to be the gold standard in terms of accurate dose calculation. However, due to the small number of validation cases, persistent uncertainties concerning material segmentation (all studies published to date have assigned human tissues data to animals due to lack of data on animal tissue compositions) and small animal positioning (particularly in the thorax with respiratory motion), major uncertainties persist and future studies are necessary to assess the accuracy of our simulation tool.

\section{v. Conclusion}

The field of very high dose-rate radiobiology is still in its infancy, but recent preclinical data showing a reduction of normal tissue toxicity, while still maintaining the same tumour control could dramatically transform the field of radiation oncology in the event of translation to clinical practice. Metrological and simulation tools are a prerequisite for the comparison of radiobiological effectiveness of different irradiation modalities. However, FLASH irradiation raises significant dosimetric challenges, and, to our knowledge, validation of Monte-Carlo modelling to describe this type of irradiation has never been reported. In this work, we modelled a prototype electron beam linear accelerator by comparing measurements and simulations, and computed the 3D dose distributions and dose-volume histograms in lungs on small animals. The results of this work, performed on a prototype 
electron linear accelerator, and more generally applicable to many particle accelerators, could support the analysis of radiobiological experiments for preclinical research with very high dose-rate electron beams.

\section{Additional information and declarations}

The authors have no conflicts of interest to disclose. This work was supported by SIRIC Institut Curie [grant $n^{\circ}$ INCa-DGOS- 4654] and the Nanotherad IDEX (Paris-Saclay University).

\section{REFERENCES}

[1] Favaudon $\mathrm{V}$ et al.Ultrahigh dose-rate FLASH irradiation increases the differential response between normal and tumor tissue in mice.SciTransl Med. 2014;6;245:ra93.

[2] Montay-Gruel P et al.Irradiation in a flash: Unique sparing of memory in mice after whole brain irradiation with dose rates above 100 Gy/s.RadiotherOncol.2017;124:365-369.

[3] Sørensen B.S, Vestergaard A, Overgaard Jet al. Dependence of cell survival on instantaneous dose rate of a linear accelerator.RadiotherOncol.2011;101(1):223-225.

[4] Auer S, Hable V, Greubel C et al. Survival of tumor cells after proton irradiation with ultra-high dose rates. RadiotherOncol. 2011;6:139.

[5] Rafat M, Bazalova M, Palma B.A et al.Impact of Very Rapid Irradiation on Clonogenic Survival. Int J RadiatOncolBiol Phys 2014;90:S790.

[6] Schuler E, Trovati S, King G, Lartey F, Rafat M, Villegas M. et al. Experimental platform for ultra-high dose rate FLASH irradiation of small animals using a clinical linear accelerator. Int J Radiat Oncol Biol Phys 2017; 97: 195-203

[7] Lempart M, Blad B, Adrian G, Bäck S, Knöös T, Ceberg C, Petersson K. Modifying a clinical linear accelerator for delivery of ultra-high dose rate irradiation, Radiotherapy and Oncology, 2019; S0167-8140(19)30059-3

[8] Karsch L et al. Dose rate dependence for different dosimeters and detectors: TLD, OSL, EBT films, and diamond detectors. Med Phys.2012;39(5):2447-55.

[9] Burns D. T. and McEwen M. R. Ion recombination corrections for the NACP parallel-plate chamber in a pulsed electron beam. Phys. Med. Biol. 1998;43(8), 2033.

[10] Chao T. C. The evaluation of 6 and $18 \mathrm{MeV}$ electron beams for small animal irradiation. Phys Med Biol.2009; 54(19):5847-60.

[11] Das T. N, Ghanty T. K and Pal H. Reactions of methyl viologen dication (MV2+) with $\mathrm{H}$ atoms in aqueous solution: Mechanism derived from pulse radiolysis measurements and ab initio MO calculations. J Phys Chem. A 2003;107:5998-6006.

[12] Katsumura Y. Ion beam pulse radiolysis study on intra-track reactions in aqueous solutions. Res Chem Intermediates 2001;27(4-5):333-341.

[13] Jaccard $M$, Petersson K, Buchillier $T$ et al. High dose-per-pulse electron beam dosimetry: Usability and dose-rate independence of ebt3 gafchromic films. Med Phys. 2017;44:725-735.

[14] Lechner W et al. Characteristic of EBT-XD and EBT3 radiochromic film dosimetry for photon and proton beams. Phys Med Biol. 2018;63(6).

[15] Sipilä P et al. Gafchromic EBT3 film dosimetry in electron beams - energy dependence and improved film read-out. J Appl Clin Med Phys.2016;17(1):360-373.

[16] Favaudon V., Tourbe, H., Houee-Levin C. and Lhoste J. M. Carboxyl radical induced cleavage of disulfide bonds in proteins. A gamma-ray and pulse radiolysis mechanistic investigation. Biochemistry. 1990;29(49), 10978-10989. 
[17] Verhaegen F and Seuntjens J Monte Carlo modelling of external radiotherapy photon beams. Phys. Med. Biol.. 2003;48(21), R107.

[18] Kapur A, Ma CM, Mok EC, Findley DO and Boyer AL. Monte Carlo calculations of electron beam output factors for a medical linear accelerator. Phys. Med. Biol. 1998;43(12), 3479.

[19] Lee CC, Chen AM, Tung CJ and Chao TC. Monte Carlo simulation of small field electron beams for small animal irradiation. Radiation Measurements. 2011;46(12), 2003-2005.

[20] Jan S. et al. GATE - Geant4 Application for Tomographic Emission: a simulation toolkit for PET and SPECT. Phys Med Biol. 2004;49(19):4543-4561.

[21] Sarrut D et al. A review of the use and potential of the GATE Monte-Carlo code for radiation therapy and dosimetry applications. Med Phys. 2014;41(6):064301.

[22] http://www.scipy.org/; 2018 [accessed 2018-11-05].

[23] Khachonkham S, Dreindl R, Heilemann G, Lechner W, Fuchs H, Palmans H, Georg D, Kuess P. Characteristic of EBT-XD and EBT3 radiochromic film dosimetry for photon and proton beams. Phys Med Biol. 2018;63(6):065007.

[24] Muir B. R and Rogers D. W. O. Monte-Carlo calculations of electron beam quality conversion factors for several ion chamber types. Med Phys2014;41:111701.

[25] Sorriaux J et al. Evaluation of gafchromic EBT3 films characteristics in therapy photon, electron and proton beams. Phys Med. 2013;29:599-606.

[26] Barouky JE et al. Practical use of Gafchromic EBT films in electron beams for in-phantom dose distribution measurements and monitor units verification, Phys Med. 2011;27(2):81-8.

[27] Russo S, Mirandola A, Molinelli S et al. Characterization of a commercial scintillation detector for 2-D dosimetry in scanned proton and carbon ion beams. Phys Med. 2017;34:48-54.

[28] Wong J et al. A high resolution small animal radiation research platform (SARRP) with x-ray tomographic guidance capabilities. Int J Radiat Oncol Biol Phys. 2008;71(5):1591-1599.

[29] Schneider U, Pedroni E and Lomax A. The calibration of CT Hounsfield units for radiotherapy treatment planning. Phys Med Biol. 1996;41(1):111.

[30] Schneider W, Bortfeld T and Schlegel W. Correlation between CT numbers and tissue parameters needed for Monte-Carlo simulations of clinical dose distributions. Phys Med Biol.2000;45(2):459.

[31] Low DA et al. A technique for the quantitative evaluation of dose distributions. Med Phys. 1998;25(5):656-661.

[32] Geant4 User's Guide for Application Developers, version: geant4 10.3 - 2016. http://geant4-userdoc.web.cern.ch/geant4userdoc/UsersGuides/ForApplicationDeveloper/BackupVersions/V10.3/fo/BookForAppliDev.pdf

[33] Smekens F, Létang JM, Noblet C, Chiavassa S, Delpon G, Freud N, Rit S and Sarrut D. Split exponential track length estimator for Monte-Carlo simulations of small-animal radiation therapy. Phys. Med. Biol. $2014 ; 59$ : 7703-7715

[34] JCL Chow, MKK Leung. Treatment planning for a small animal using Monte Carlo simulation Medical Physics 2007 ;34, 4810

[35] Hill MA, Vojnovic B. Implications of respiratory motion for small animal image-guided radiotherapy. Br J Radiol 2017; 90: 20160482 


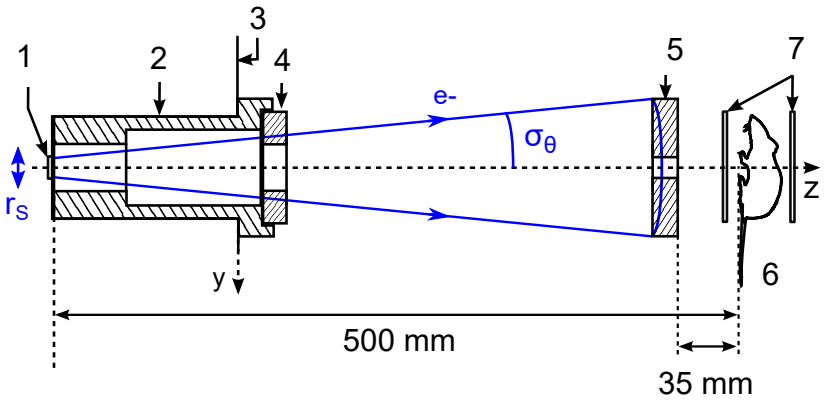




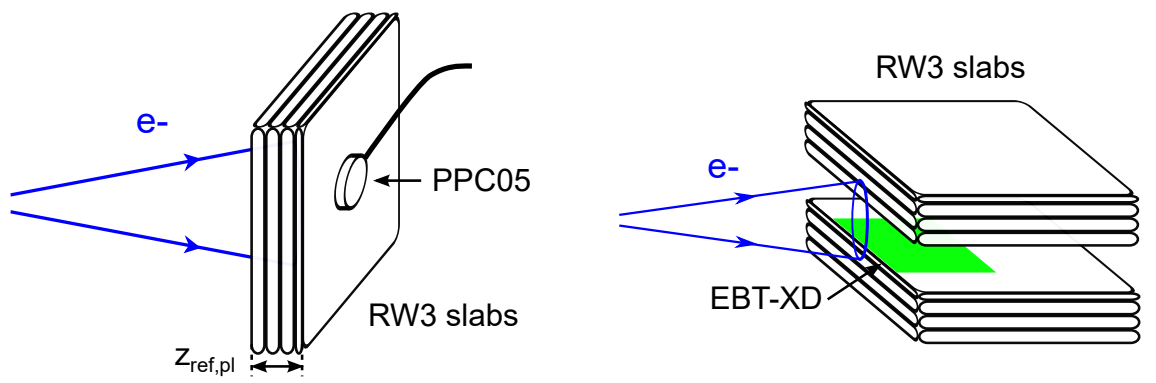




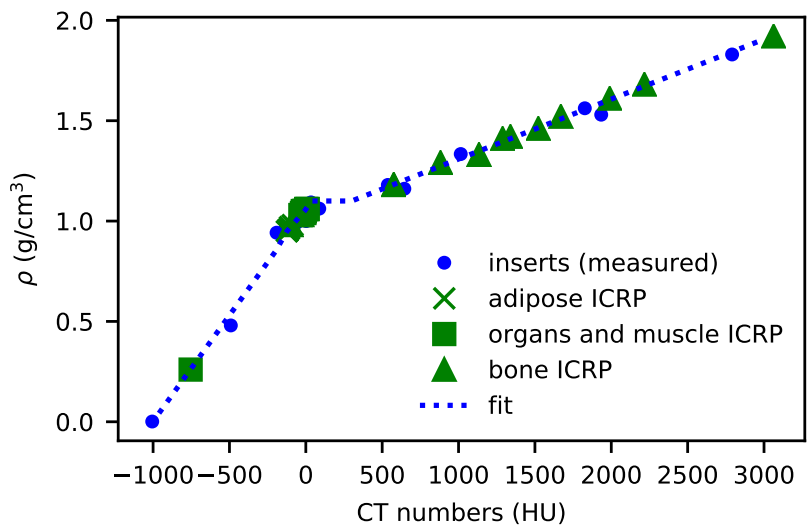




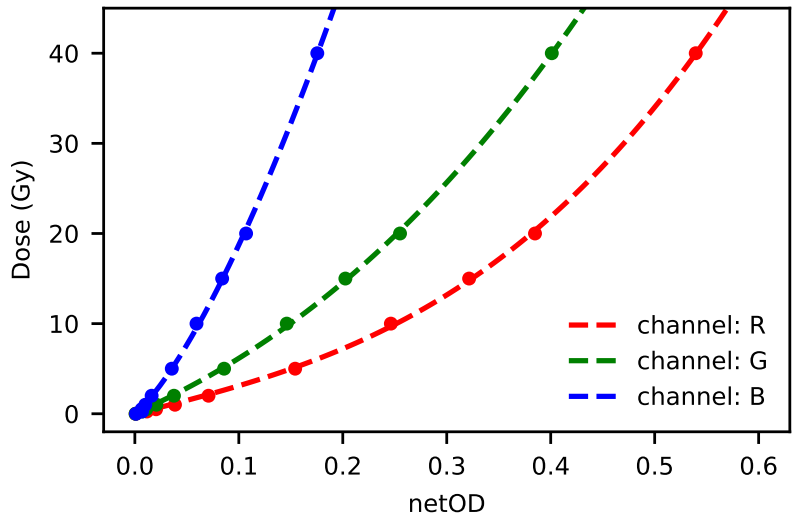




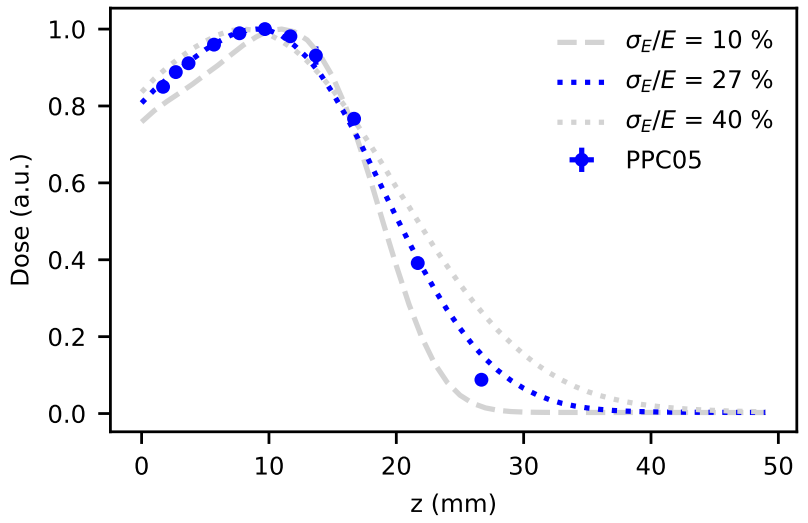




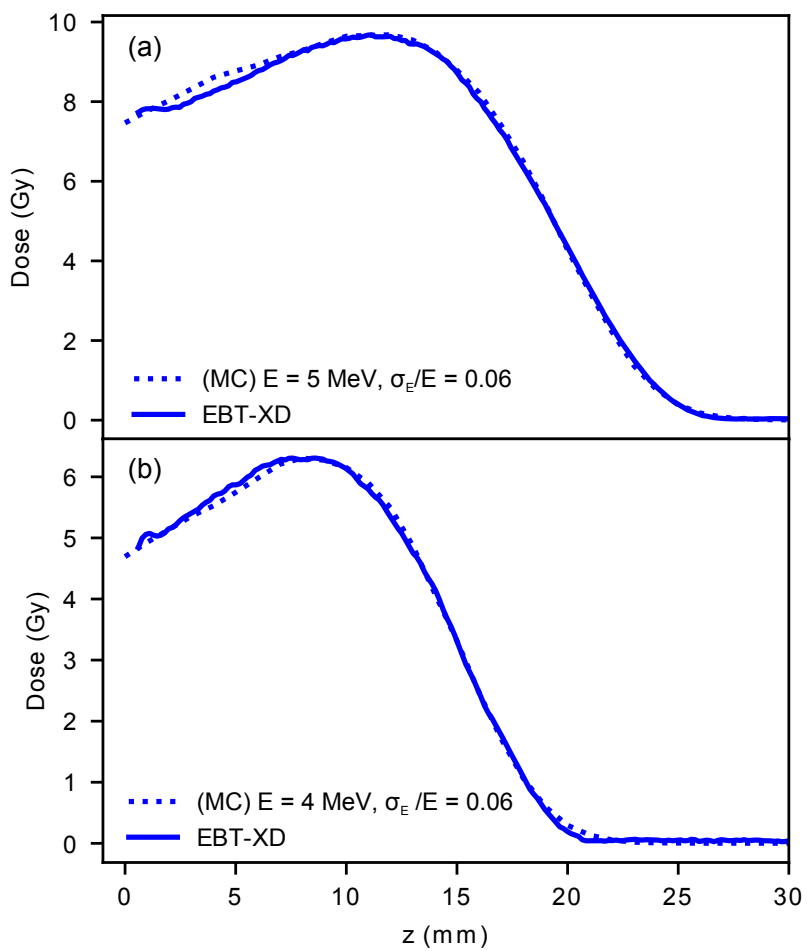



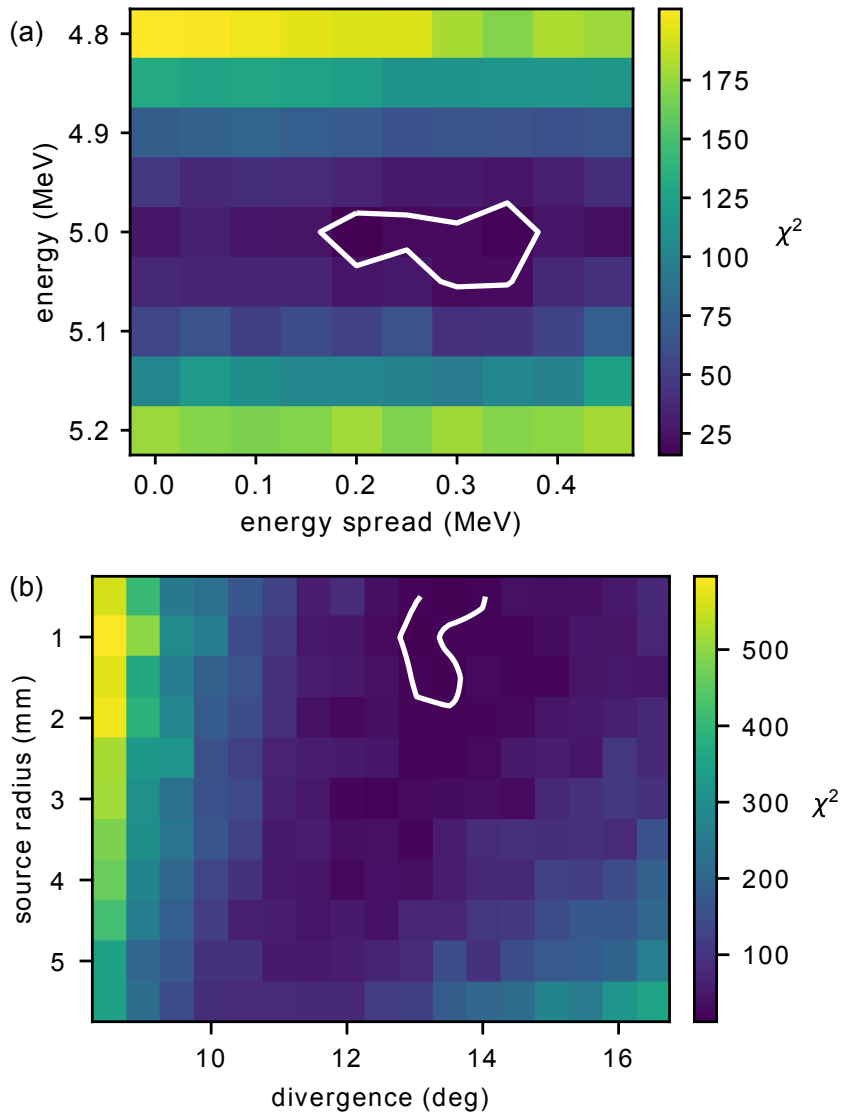


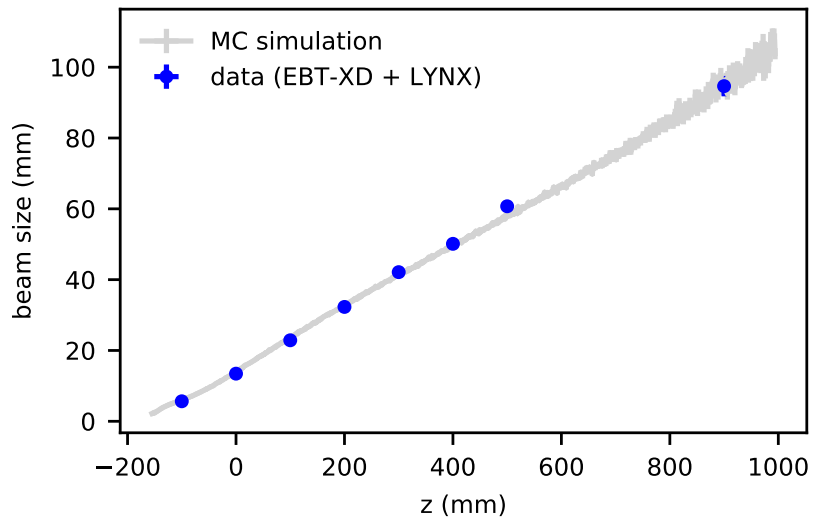



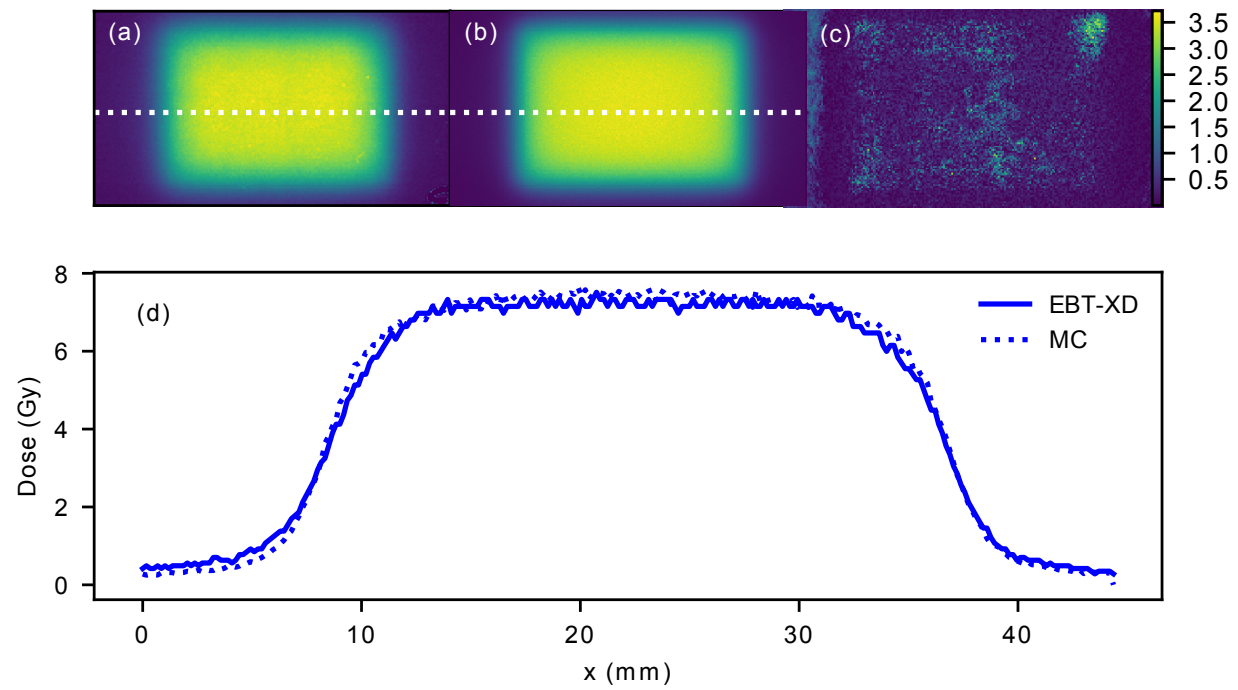

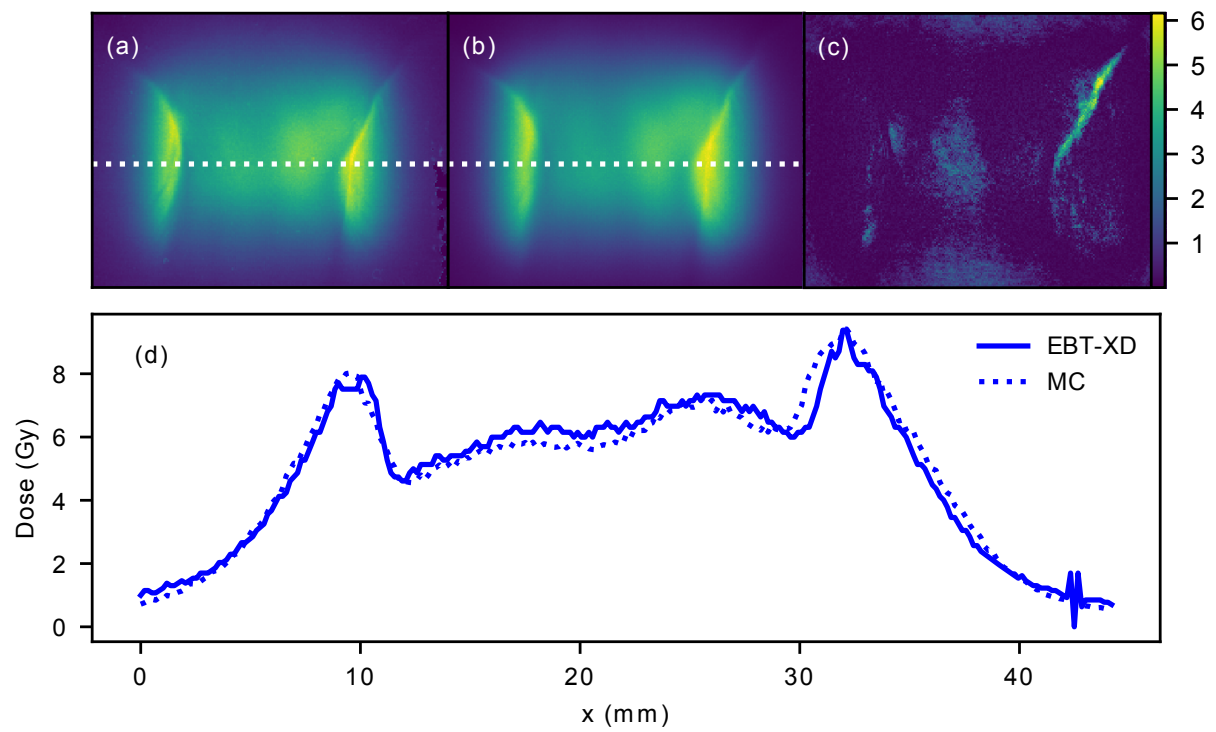
(a)

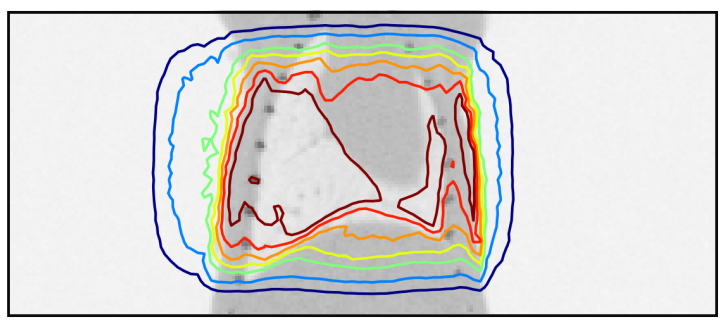

(b)
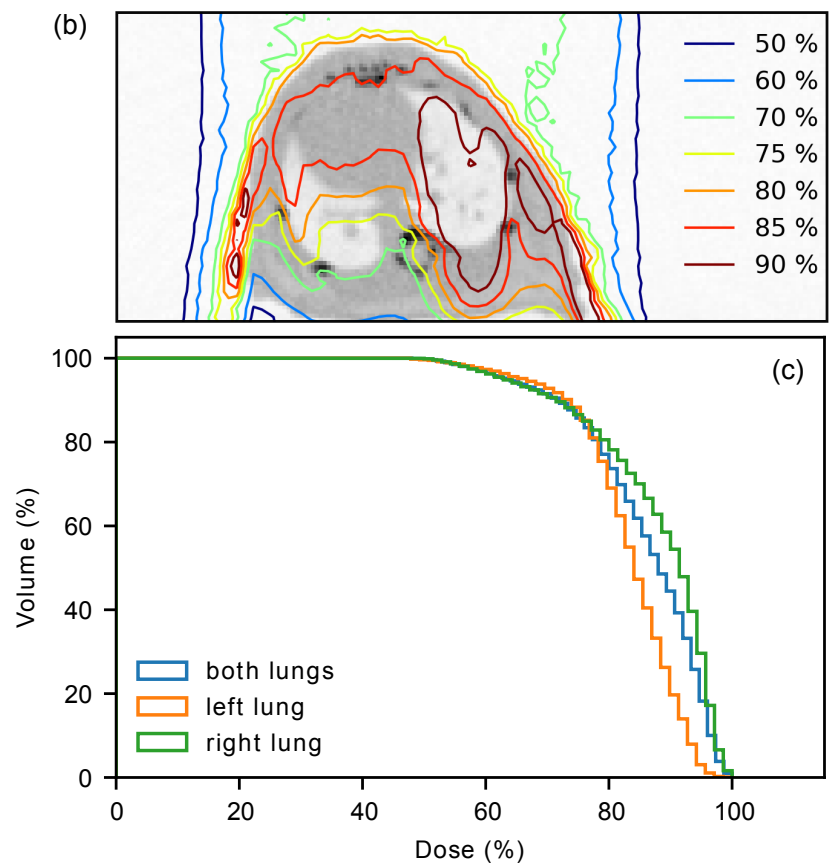

(d)

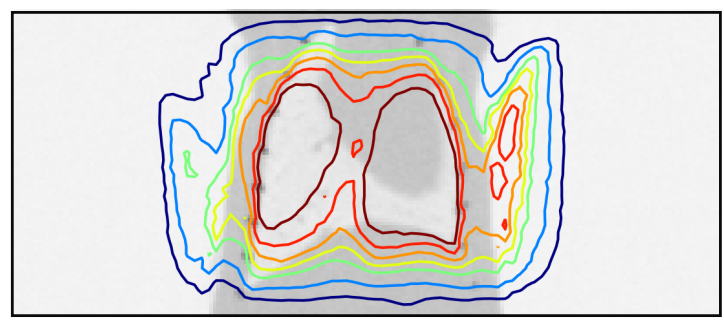

(e)
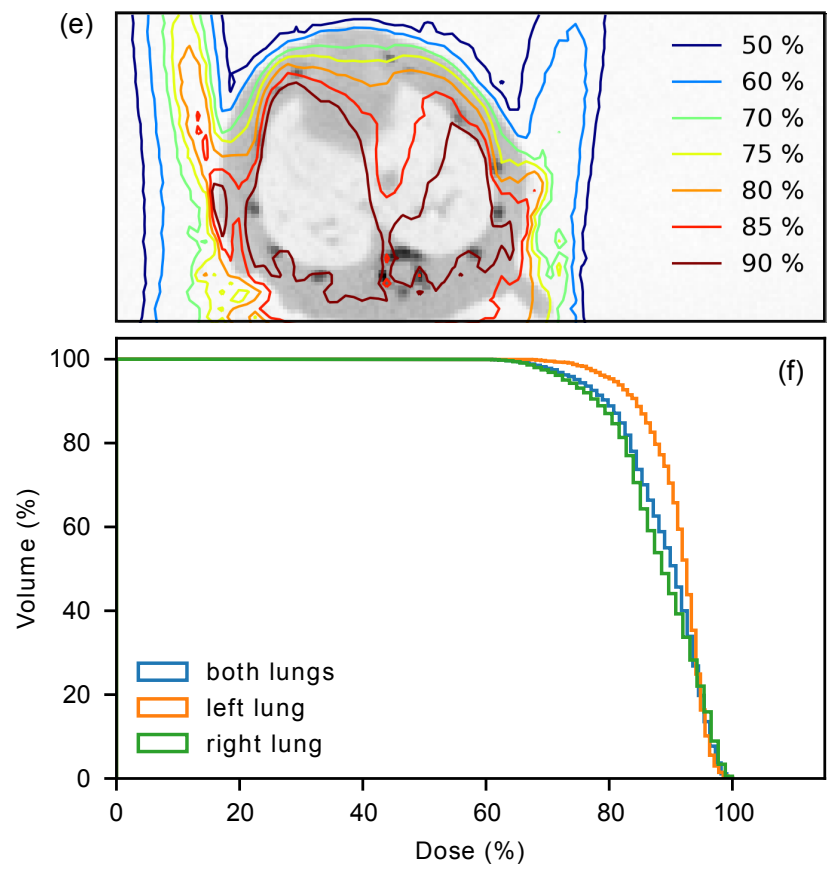